\title{
Alternative Fertility Management for Establishing New Apple Orchards in the Mid-Atlantic
}

\author{
Ashley A. Thompson \\ Department of Horticulture, Alson H. Smith, Jr. Agricultural Research \\ and Extension Center, Virginia Polytechnic Institute and State University, \\ 595 Laurel Grove Road, Winchester, VA 22602
}

\author{
Gregory M. Peck ${ }^{1}$ \\ School of Integrative Plant Science, Horticulture Section, Cornell University, \\ 121 Plant Science Building, Ithaca, NY 14853
}

Additional index words. compost, microbial ecology, nitrogen fertilizer, soil fertility

\begin{abstract}
In the Mid-Atlantic, mineral nitrogen (N) fertilizers are applied in high-density apple (Malus $\times$ domestica Borkh.) orchards to increase tree vegetative growth and achieve earlier fruiting. However, when applied in excess of plant needs, $N$ fertilizer applications are an unnecessary expense and may lead to $\mathrm{N}$ leaching and groundwater pollution. Therefore, it is necessary to develop orchard fertilization programs that simultaneously provide adequate crop nutrition and minimize $N$ loss into the environment. Nitrogen was applied in each of 3 years to newly planted 'Red Delicious cv Schlect'/ 'M.26' trees at $67 \mathrm{~kg} \mathrm{~N} / \mathrm{ha} / \mathrm{year}$ in six fertilizer treatments: 1) two equal applications of granular calcium nitrate $\left.\left[\mathrm{Ca}\left(\mathrm{NO}_{3}\right)_{2}\right] ; 2\right)$ chicken litter compost; 3) yardwaste compost; 4) a combination of chicken litter compost and granular $\mathrm{Ca}\left(\mathrm{NO}_{3}\right)_{2}$ with equal amounts of $\mathrm{N}$ from each fertilizer; 5) a combination of yardwaste compost and granular $\mathrm{Ca}\left(\mathrm{NO}_{3}\right)_{2}$ with equal amounts of $\mathrm{N}$ from each fertilizer; and 6) fertigation which consisted of eight weekly applications of solubilized $\mathrm{Ca}\left(\mathrm{NO}_{3}\right)_{2}$. Nonfertilized trees served as the control. In the third year of this experiment, the two chicken litter compost treatments had the greatest soil extractable $P$, the yardwaste compost treatment had the greatest soil extractable $\mathrm{K}$, both full-rate compost treatments had greater soil extractable Mg than the other treatments, and all four compost treatments had greater soil extractable Mn than the treatments without compost. The four compost treatments also had greater soil extractable Ca and $B$ than treatments without compost. By the third year of the experiment, the four compost treatments also had greater soil organic matter (OM) and soil $\mathbf{C}$ (with the integrated chicken litter compost treatment having similar soil $\mathbf{C}$ to the other treatments). Potentially mineralizable nitrogen and soil microbial biomass were similar among the treatments over the course of this experiment. The full rate chicken litter compost treatment and both yardwaste compost treatments had greater soil microbial respiration in 2015. The fertigation treatment performed similarly to the treatment where $\mathrm{Ca}\left(\mathrm{NO}_{3}\right)_{2}$ was applied as a granular product to the soil. Treatment differences found for the soil properties did not translate to increased tree size or leaf $\mathbf{N}$ content, suggesting that the trees were able to acquire sufficient $N$ from the soil under all of the treatments. Our results suggest that applying fertilizers to fine textured soil with relatively high $\mathrm{OM}$ may not increase apple tree growth or productivity within the first 3 years after planting. In addition, compost applications can improve many soil properties, but these differences may not result in improved orchard productivity within 3 years.
\end{abstract}

The profitability of high-density apple (Malus $\times$ domestica Borkh.) orchards depends on rapidly establishing tree biomass and then

\footnotetext{
Received for publication 3 Mar. 2017. Accepted for publication 2 Aug. 2017.

We thank Abby Kowalski, Taylor Mackintosh, David Carbaugh, Sierra Athey, Chandler DeHaven, and Jim Warren for their assistance with this experiment, and Tony Wolf and Sam Wortman for their thoughtful comments on this article. This work was supported by the U.S. Department of Agriculture-National Institute of Food and Agriculture under Southern SARE award number LS13-258, the Virginia Agricultural Council, the Virginia Apple Research Program, the Virginia Agricultural Experiment Station, and Virginia Tech's Department of Horticulture.

${ }^{1}$ Corresponding author. E-mail: gmp32@cornell.edu.
}

(this includes the states of Delaware, Maryland, New Jersey, New York, North Carolina, Pennsylvania, Virginia, and West Virginia). In addition, there have been few studies describing the effects of carbon-based fertilizers, such as compost, on apple tree growth and productivity in newly planted highdensity orchards in this region.

Furthermore, mineral $\mathrm{N}$ fertilizers may lead to negative environmental impacts. For example, ground applications of mineral $\mathrm{N}$ fertilizers in orchards have been observed to increase $\mathrm{N}$ leaching in orchard systems (Dong et al., 2005b; Merwin et al., 1996). This is of growing concern in watersheds, such as the Chesapeake Bay, where agricultural $\mathrm{N}$ has become an environmental pollutant. In an effort to reduce $\mathrm{N}$ pollution, the Environmental Protection Agency has enacted Total Maximum Daily Load limits on the amount of $\mathrm{N}$ that may enter the Chesapeake Bay Watershed (United States Environmental Protection Agency, 2010). Because of these regulations, detailed nutrient management plans are now needed for animal and agronomic productions systems within Chesapeake Bay watershed. It is possible that horticultural crop production systems may need to develop similar nutrient management plans in the future. Given these challenges, it is important to develop fertilizer application approaches and materials that can reduce environmental $\mathrm{N}$ loss from apple orchards.

One approach to improving soil fertility and reducing $\mathrm{N}$ loss is to use carbon-based amendments, such as composts. In apple orchards, compost applications have been shown to improved edaphic properties, including soil OM, microbial biomass carbon (C), microbial respiration, and soil mineral nutrition in fine- and coarse-textured soils (Forge et al., 2013; Neilsen et al., 2014; Rumberger et al., 2004; Sas-Paszt et al., 2014; Yao et al., 2006). For example, Kramer et al. (2006) observed that compost amended soils had $42 \%$ greater $\mathrm{OM}$ and 35 and $57 \%$ greater microbial biomass $\mathrm{C}$ and $\mathrm{N}$, respectively, than soil fertilized with mineral $\mathrm{N}$ fertilizer. In addition, compost applications in coarse soil increased apple leaf $\mathrm{N}$ by $5 \%, \mathrm{~K}$ by $4 \%$, Mn by $13 \%$, and $\mathrm{Zn}$ by $5 \%$ compared with an unfertilized control (Sas-Paszt et al., 2014). However, compost applications do not appear to affect the vegetative growth or fruit yield or quality independent of soil texture (Forge et al., 2013; Neilsen et al., 2014; Rumberger et al., 2004; Sas-Paszt et al., 2014; Yao et al., 2006). In contrast to the results observed in apple orchards, Baldi et al. (2010) found that when compost was tilled into fine textured soil to a depth of $25 \mathrm{~cm}$ in a 7-year-old peach orchard, fruit yield increased by $38 \%$ in the first year, as did soil quality measures such as, OM, soil mineral nutrition, and microbial biomass $\mathrm{C}$ compared with the unfertilized control.

It appears that the contrasting results are contingent on soil texture, plant species, and the specific feedstock used to produce the compost. Composts made exclusively from 
plant material, such as yard wastes, have a higher $\mathrm{C}: \mathrm{N}$ ratio than manure-based composts. Amending soil with a high $\mathrm{C}: \mathrm{N}$ ratio compost will initially immobilize $\mathrm{N}$, then mineralize $\mathrm{N}$ slowly but for a longer period of time than manure-based composts (Hartz et al., 2000). Therefore, it is necessary to determine how compost produced from different feedstocks, such as chicken litter and yard waste, may affect tree growth and productivity in an orchard.

Using an integrated compost-mineral fertilizer strategy may provide trees with $\mathrm{N}$ when it is most needed, while also conferring the benefits of compost mentioned previously. In an apple orchard with coarse textured soils, integrated compost-mineral fertilizer applications resulted in 33\% more soil $\mathrm{OM}$ and $55 \%$ less $\mathrm{NO}_{3}{ }^{-}$leaching than soil fertilized with mineral fertilizer $\left[\mathrm{Ca}\left(\mathrm{NO}_{3}\right)_{2}\right]$ (Kramer et al., 2006). However, integrated compost-mineral fertilizer applications did not increase soil microbial biomass $\mathrm{C}$ and $\mathrm{N}$ or potentially mineralizable $\mathrm{N}$ compared with the mineral fertilizer treatment. In other horticultural cropping systems, integrated fertilization approaches have been used to increase crop yields while improving soil quality. For example, parsley plants that were fertilized with the integrated treatment, compost and $\mathrm{NH}_{4} \mathrm{NO}_{3}$, had $69 \%$ more biomass than an unfertilized control, whereas plants that received compost alone increased biomass by $18 \%$ compared with an unfertilized control (Mylavarapu and Zinati, 2009). In this study, the integrated treatments also increased soil total $\mathrm{C}$ and $\mathrm{N}$, microbial biomass $\mathrm{C}$, and microbial respiration similarly to the compost amendments. Similar results have also been documented for broccoli and tomatoes planted in coarse-textured soils (Hernández et al., 2014; Stamatiadis et al., 1999). Thus, integrated compost-mineral fertilizer applications may be an approach to reduce environmental $\mathrm{N}$ loss while providing trees with sufficient $\mathrm{N}$ and enhancing soil quality.

Fertigation, a method which dispenses soluble fertilizers through irrigation lines, is another strategy that may reduce the negative environmental impacts from mineral fertilizer applications. Studies in arid apple producing regions with coarse soil, such as Israel and British Columbia, CAN, have demonstrated that fertigation can improve fruit quality and yield, tree growth, and leaf $\mathrm{N}$ concentration (Dong et al., 2005b; Klein et al., 1989; Neilsen et al., 2009). In a pot culture study, fertigating trees with $\mathrm{N}$ increased shoot growth by $\approx 58 \%$ and $82 \%$ compared with foliar $\mathrm{N}$ applications and the unfertilized control, respectively, and increased fruit yield by $28 \%$, and fruit size by $22 \%$ compared with the unfertilized control (Dong et al., 2005a, 2005b). Similar results were observed in temperate regions with fertile, fine-textured clay soils, where $\mathrm{N}$ fertigation increased fruit yield by $25 \%$, flower bud formation by $40 \%$, and shoot growth by $85 \%$ in established apple orchards compared with broadcast fertilizer use (Kipp,
1992). Results from this study suggest that fertigation may improve apple tree yield and shoot growth in the fine-textured soils of the Mid-Atlantic.

The objectives of our study were to evaluate the effects of fertilizers, including ground applied $\mathrm{Ca}\left(\mathrm{NO}_{3}\right)_{2}$, compost, integrated compost-Ca( $\left(\mathrm{NO}_{3}\right)_{2}$, and fertigation with $\mathrm{Ca}\left(\mathrm{NO}_{3}\right)_{2}$, on tree growth and productivity and soil fertility in a newly planted apple orchard. We hypothesized that the integrated applications of composts and $\mathrm{Ca}\left(\mathrm{NO}_{3}\right)_{2}$ would supply apple trees with sufficient nitrogen to maintain adequate growth and productivity while also improving soil quality.

\section{Materials and Methods}

The experiment occurred on a Poplimento silt loam, a fine, mixed, subactive, mesic Ultic Hapludalf soil [Natural Resource Conservation Service (NRCS), 2001]. In Apr. 2013, three rows of 49 'Red Delicious cv Schlect'/'Malling 26' (M.26) trees were planted at a spacing of $1.5 \mathrm{~m}$ between trees and $4.5 \mathrm{~m}$ between rows at the Virginia Tech Alson H. Smith, Jr. Agricultural Research and Extension Center in Winchester, VA $\left(39^{\circ} 06^{\prime} \mathrm{N}, 78^{\circ} 17^{\prime} \mathrm{W}\right)$. All trees were trained as a vertical-axis on a single-wire trellis and were uniformly treated for crop damaging arthropods, diseases, and weeds according to regional recommendations (Pfeiffer et al., 2015). Irrigation was supplied through drip irrigation tubing with in-line emitters. In 2013 and 2014, all flower clusters were removed by hand to prevent fruit set and encourage vegetative growth. On 20 May 2015, fruitlets were thinned to three fruit per trunk cross-sectional area (TCSA) on each tree to prevent overcropping young trees (Robinson et al., 2013).

Treatments were replicated four times in a randomized complete block design using five-tree sets for each experimental unit. The two end trees in each five-tree set served as buffers and were not used for data collection. Fertilizer treatments were applied to the soil on 13 May 2013, 19 May 2014, and 14 May 2015. Before treatment applications, there were no significant differences in soil $\mathrm{pH}$, OM, CEC, or Mehlich 1 extractable mineral nutrient among the treatment plots. All treatments, except the control, were applied at a rate of $67 \mathrm{~kg}$ of plant available N/ha/year. Fertilizer treatments included: 1) an unfertilized control $(\mathrm{CON}), 2)$ a split application of water soluble calcium nitrate $\left[\mathrm{Ca}\left(\mathrm{NO}_{3}\right)_{2}\right]$ (MIN) (Yara, Oslo, Norway), 3) chicken litter (CL) compost, 4) yard waste (YW) compost, 5) a combination of chicken litter compost and $\mathrm{Ca}\left(\mathrm{NO}_{3}\right)_{2}(\mathrm{CL}+\mathrm{MIN})$ with equal amounts of $\mathrm{N}$ from each fertilizer, 6) a combination of yard waste compost and $\mathrm{Ca}\left(\mathrm{NO}_{3}\right)_{2}(\mathrm{YW}+\mathrm{MIN})$ with equal amounts of $\mathrm{N}$ from each fertilizer, and 7) hand fertigation with $\mathrm{Ca}\left(\mathrm{NO}_{3}\right)_{2}$ (FGN) for eight weeks beginning in May and ending in July of each year. The first split application of the MIN treatment occurred on 1 June 2013, 28 May 2014, and 28 May 2015, and the second application of MIN occurred on 2 July 2013, 2014, and 2015. Compost was spread by hand from the base of the trunk to the edge of the vegetation free strip $(\approx 83 \mathrm{~cm}$ from the trunk), and $\mathrm{Ca}\left(\mathrm{NO}_{3}\right)_{2}$ was applied around the base of the trees. The amounts of other mineral nutrients and OM differed among the applied treatments. Before compost application each year, compost nutrient analysis was performed by the Penn State Agricultural Analytical Services Laboratory (University Park, PA) in accordance with standard compost testing methods described by the United States Department of Agriculture and United States Composting Council (2002). Plant available $\mathrm{N}$ application rates were calculated, and equalized in all treatments (Table 1). Plant available $\mathrm{N}$ was calculated as the sum of $10 \%$ the organic $\mathrm{N}$ plus the inorganic $\mathrm{N}$ $\left(\mathrm{NH}_{4}-\mathrm{N}\right.$ and $\left.\mathrm{NO}_{3}-\mathrm{N}\right)$ (Campbell-Nelson, 2015).

Trunk cross-sectional area was measured $30 \mathrm{~cm}$ above the graft union at the time of planting and when trees were dormant in all subsequent years. Central leader length was measured from the end of the previous year's growth to the topmost terminal bud when trees were dormant each year. On 14 Sept. 2015 , trees were harvested in their entirety and we recorded total fruit number and weight per tree. Four fruit were randomly selected from each of the three sample trees and used to determine fruit quality and maturity. Fruit size, weight, and flesh firmness were measured using computer integrated calipers, balance, and penetrometer, respectively (Fruit Texture Analyzer; GÜSS Manufacturing Ltd., Strand, South Africa). Flesh firmness was measured once on each side of the fruit after removing part of the peel with an $11.1 \mathrm{~mm}$ diameter tip. Starch pattern index was visually assessed using the Cornell Starch-Iodine Index (Blanpied and Silsby, 1992). Peel color was visually assessed as the percentage of the apple surface that was red. Fruit internal ethylene concentration was measured from $1 \mathrm{~mL}$ of gas drawn from the apple cortex using a $7890 \mathrm{~A}$ gas chromatograph (Agilent Technologies, Santa Clara, CA). The soluble solid concentration was measured using a refractometer (PAL-1; Atago, Tokyo, Japan). Flower clusters were counted on 28 Apr. 2015 and 16 Apr. 2016.

On 14 Aug. 2013, 22 Aug. 2014, and 11 Aug. 2015, 20 leaves per sample tree (60 per experimental unit) were removed from the middle of the current year's branch growth, dried in an oven at $80{ }^{\circ} \mathrm{C}$ for $3 \mathrm{~d}$, and measured for mineral concentration at the Penn State Agricultural Analytical Services Laboratory (University Park, PA). Leaf N concentration was measured using the combustion analysis method (Horneck and Miller, 1998) on a Vario Max N/C analyzer (Elementar, Hanau, Germany). Leaf P, K, Ca, $\mathrm{Mg}, \mathrm{B}, \mathrm{Cu}$, and $\mathrm{Zn}$ were measured using a 730-ES ICP Optical Emission Inductively Coupled Plasma (OES-ICP) Spectrometer 
(Agilent Technologies, Santa Clara, CA) after dry-ashing (Miller, 1998).

On 8 Sept. 2013, 3 Sept. 2014, and 24 Aug. 2015, soil samples were collected $30 \mathrm{~cm}$ from the trunk on the north, south, east, and west sides of the center experimental tree to a depth of $15 \mathrm{~cm}$ using a 7-cm diameter soil auger. Soil was placed in a bag, homogenized by hand, sieved (U.S. number 10 soil sieve; $2 \mathrm{~mm}$ mesh) and then stored at $4{ }^{\circ} \mathrm{C}$ until use in biological assays. A subsample of soil samples was sent to the Virginia Tech Soil Testing Laboratory (Blacksburg, VA) for analysis of plant available nutrients, $\mathrm{pH}$, and CEC. Before soil physiochemical analysis, soil samples were air dried and crushed with a hammer mill-type crushing machine (Agvise, Benson, MN). Mehlich 1 solution was used to extract $\mathrm{P}, \mathrm{K}, \mathrm{Ca}, \mathrm{Mg}, \mathrm{Zn}, \mathrm{Mn}, \mathrm{Cu}$, $\mathrm{Fe}$, and $\mathrm{B}$ from $4 \mathrm{~cm}^{3}$ of soil. The mineral nutrient concentration was determined from the extraction by OES-ICP (Acros Spectro, Mahwah, NJ). Cation exchange capacity was estimated by summation of the non-acidgenerating cations $(\mathrm{Ca}, \mathrm{Mg}$, and $\mathrm{K})$ and Mehlich 1 soil-buffer acidity. Soil $\mathrm{pH}$ was measured using a $\mathrm{pH}$ meter (WP-80D; TPS Pty Ltd., Springwood, Australia) fitted with a combination $\mathrm{pH}$ electrode (Orion model 8165BNWP Ross Sure-Flow; ThermoFisher, Waltham, MA). Total $\mathrm{C}$ and total $\mathrm{N}$ were measured at the Cornell Nutrient Analytical Laboratory on a CHN Elemental Analyzervario EL (Elementar, Hanau, Germany) after soil was ground to a fine powder using a mortar and pestle. Soil OM and soluble salts (SS) were measured at the Virginia Tech Soil Testing Laboratory (Blacksburg, VA) in 2013 and 2014, and at the Cornell Nutrient Analytical Laboratory (Ithaca, NY) in 2015. At the Virginia Tech Soil Testing Laboratory, soil OM was measured after loss on ignition

Table 1. The carbon to nitrogen ratio $(\mathrm{C}: \mathrm{N})$, organic matter $(\mathrm{OM})$, total $\mathrm{C}$, organic $\mathrm{N}$, nitrogen as ammonium $\left(\mathrm{NH}_{4}-\mathrm{N}\right)$, nitrogen as nitrate $\left(\mathrm{NO}_{3}-\mathrm{N}\right)$, phosphorus $(\mathrm{P})$, and potassium $(\mathrm{K})$ content of the chicken litter (CL) and yard waste (YW) composts applied in 2013, 2014, and 2015 to 'Red Delicious' apple trees in Winchester, VA.

\begin{tabular}{lcccccrrr}
\hline Compost & $\mathrm{C}: \mathrm{N}$ & $\begin{array}{c}\mathrm{OM} \\
\left(\mathrm{g} \cdot \mathrm{kg}^{-1}\right)\end{array}$ & $\mathrm{C}\left(\mathrm{g} \cdot \mathrm{kg}^{-1}\right)$ & $\begin{array}{c}\text { Organic } \\
\mathrm{N}\left(\mathrm{g} \cdot \mathrm{kg}^{-1}\right)\end{array}$ & $\begin{array}{c}\mathrm{NH}_{4}-\mathrm{N} \\
\left(\mathrm{mg} \cdot \mathrm{kg}^{-1}\right)\end{array}$ & $\begin{array}{c}\mathrm{NO}_{3}-\mathrm{N} \\
\left(\mathrm{mg} \cdot \mathrm{kg}^{-1}\right)\end{array}$ & $\mathrm{P}\left(\mathrm{g} \cdot \mathrm{kg}^{-1}\right)$ & $\mathrm{K}\left(\mathrm{g} \cdot \mathrm{kg}^{-1}\right)$ \\
\hline $2013 \mathrm{CL}$ & 15.8 & 458 & 260 & 16.5 & 5 & 1,012 & 18.8 & 7.5 \\
$2013 \mathrm{YW}$ & 14.4 & 538 & 246 & 17.1 & 56 & 21 & 4.6 & 11.5 \\
$2014 \mathrm{CL}$ & 14.9 & 475 & 293 & 19.6 & 5 & 501 & 19.0 & 8.6 \\
$2014 \mathrm{YW}$ & 18.8 & 608 & 342 & 18.1 & 37 & 44 & 4.1 & 11.2 \\
$2015 \mathrm{CL}$ & 12.9 & 473 & 246 & 19.0 & 5 & 601 & 16.8 & 6.5 \\
$2015 \mathrm{YW}$ & 18.1 & 515 & 291 & 16.1 & 48 & 92 & 3.5 & 7.8 \\
\hline
\end{tabular}

(LOI) at $360{ }^{\circ} \mathrm{C}$ for $2 \mathrm{~h}$ (Blue $\mathrm{M}$ model $\mathrm{CW}$ 6680F; New Columbia, PA), and an EC probe was used to measure SS (3100 Conductivity Instrument; YSI, Yellow Springs, OH). At the Cornell Nutrient Analytical Laboratory, soil OM was measured using the LOI method at $500{ }^{\circ} \mathrm{C}$ for $2 \mathrm{~h}$, and $\mathrm{SS}$ were measured using an electrical conductivity (EC) probe (Symphony SB70C Conductivity Meter; VWR, Radnor, PA).

Soil respiration was measured using the method described by Rodella and Saboya (1999). Briefly, $50 \mathrm{~g}$ of soil was placed in an air-tight jar with a vial containing $20 \mathrm{~mL}$ of $0.5-\mathrm{M} \mathrm{NaOH}$ solution to trap the evolved $\mathrm{CO}_{2}$ gas. The $\mathrm{EC}$ of the $\mathrm{NaOH}$ solution was measured weekly for 6 weeks using an EC meter (model 2052; Amber Science Inc., Eugene, OR). The EC of each sample was compared with a blank containing $50 \mathrm{~g}$ of autoclaved, dried sand, and to a $\mathrm{CO}_{2}$ saturated standard of 0.25 -M sodium bicarbonate $\left(\mathrm{Na}_{2} \mathrm{CO}_{3}\right)$.

Potentially mineralizable nitrogen (PMN) was estimated as the difference in the $\mathrm{NH}_{4}-\mathrm{N}$ concentration before and after a 7-d incubation of $10 \mathrm{~g}$ of soil extracted in a $2 \mathrm{M} \mathrm{KCl}$ solution. The nitrate $\left(\mathrm{NO}_{3}-\mathrm{N}\right)$ concentration of each sample was also measured to ensure that anaerobic incubation had occurred. The nonincubated sample contained $40 \mathrm{~mL}$ of $2 \mathrm{M}$ $\mathrm{KCl}$ and was extracted after $1 \mathrm{~h}$ of agitation on an orbital shaker at $3.3 \mathrm{r} \cdot \mathrm{s}^{-1}$ and centrifugation at $500 g_{\mathrm{n}}$ for $10 \mathrm{~min}$ before filtration

Table 2. Tree size, yield, and fruit maturity and quality of 'Red Delicious'/'M.26' trees and fruit under the unfertilized control (CON) and six fertilizer treatments [calcium nitrate (MIN), chicken litter compost (CL), yard waste compost (YW), integrated chicken litter compost and calcium nitrate (CL + MIN), integrated yard waste compost and calcium nitrate (YW + MIN), and fertigation (FGN)] in Winchester, VA. Trunk cross-sectional area (TCSA) and central leader growth were measured on 3 Dec. 2015; fruit weight, flesh firmness, starch pattern index (SPI), peel color, soluble solids concentration (SSC), and internal ethylene concentration (IEC) were determined from twelve fruit per experimental unit harvested on 14 Sept. 2015.

\begin{tabular}{|c|c|c|c|c|c|c|c|c|c|}
\hline Treatment & $\operatorname{TCSA}\left(\mathrm{cm}^{2}\right)$ & $\begin{array}{c}\text { Leader } \\
\text { growth }(\mathrm{cm})\end{array}$ & Yield (kg/tree) & Fruit wt (g) & $\begin{array}{c}\text { Flesh } \\
\text { firmness }(\mathrm{N})\end{array}$ & SPI (1-8) & Peel color $(\%)$ & $\mathrm{SSC}\left({ }^{\circ}\right.$ Brix $)$ & $\operatorname{IEC}\left(\mu \mathrm{L} \cdot \mathrm{L}^{-1}\right)$ \\
\hline$\overline{\mathrm{CON}}$ & 11.8 & 49.4 & $6.7 \mathrm{~A}^{\mathrm{z}}$ & 276 & 68.9 & 4.3 & $94 \mathrm{AB}$ & 14.1 & 22.1 \\
\hline MIN & 11.7 & 53.2 & $5.7 \mathrm{~A}$ & 287 & 68.5 & 4.3 & $95 \mathrm{~A}$ & 14.6 & 26.5 \\
\hline CL & 11.0 & 58.3 & $3.3 \mathrm{~B}$ & 269 & 68.9 & 4.6 & $94 \mathrm{AB}$ & 13.9 & 20.2 \\
\hline YW & 11.5 & 55.1 & $5.4 \mathrm{AB}$ & 277 & 68.0 & 4.5 & $93 \mathrm{AB}$ & 14.3 & 20.2 \\
\hline $\mathrm{CL}+\mathrm{MIN}$ & 11.2 & 49.2 & $5.1 \mathrm{AB}$ & 262 & 68.0 & 4.3 & $94 \mathrm{AB}$ & 13.8 & 18.4 \\
\hline $\mathrm{YW}+\mathrm{MIN}$ & 11.6 & 57.9 & $5.6 \mathrm{~A}$ & 258 & 68.0 & 4.3 & $93 \mathrm{AB}$ & 13.9 & 23.9 \\
\hline FGN & 11.8 & 48.9 & $6.1 \mathrm{~A}$ & 255 & 69.8 & 4.3 & $92 \mathrm{~B}$ & 13.7 & 28.7 \\
\hline
\end{tabular}

${ }^{\mathrm{z} D i f f e r e n t ~ l e t t e r s ~ w i t h i n ~ a ~ c o l u m n ~ i n d i c a t e ~ s i g n i f i c a n t l y ~ d i f f e r e n t ~ m e a n s ~ a t ~} P \leq 0.05$ using Tukey's HSD test $(n=4)$.

Table 3. Leaf mineral concentration measured in 2013, 2014, and 2015 from 'Red Delicious'/'M.26' trees the unfertilized control (CON) and six fertilizer treatments [calcium nitrate (MIN), chicken litter compost (CL), yardwaste compost (YW), integrated chicken litter compost and calcium nitrate (CL + MIN), integrated yardwaste compost and calcium nitrate (YW + MIN), and fertigation (FGN)] in Winchester, VA.

\begin{tabular}{|c|c|c|c|c|c|c|c|c|}
\hline & $\mathrm{N}(\%)^{\mathrm{y}}$ & $\mathrm{P}(\%)$ & $\mathrm{K}(\%)$ & $\mathrm{Ca}(\%)$ & $\mathrm{Mg}(\%)$ & $\mathrm{B}\left(\mathrm{mg} \cdot \mathrm{kg}^{-1}\right)$ & $\mathrm{Cu}\left(\mathrm{mg} \cdot \mathrm{kg}^{-1}\right)$ & $\mathrm{Zn}\left(\mathrm{mg} \cdot \mathrm{kg}^{-1}\right)$ \\
\hline$\overline{\mathrm{CON}}$ & 2.5 & $0.171 \mathrm{AB}^{\mathrm{z}}$ & $1.68 \mathrm{~B}$ & $1.3 \mathrm{AB}$ & 0.27 & $40.5 \mathrm{C}$ & 4.5 & 222 \\
\hline MIN & 2.6 & $0.171 \mathrm{AB}$ & $1.70 \mathrm{~B}$ & $1.4 \mathrm{~A}$ & 0.28 & $41.3 \mathrm{BC}$ & 4.6 & 211 \\
\hline $\mathrm{CL}$ & 2.6 & $0.177 \mathrm{~A}$ & $1.76 \mathrm{AB}$ & $1.3 \mathrm{AB}$ & 0.28 & $44.5 \mathrm{~A}$ & 4.4 & 234 \\
\hline YW & 2.5 & $0.170 \mathrm{AB}$ & $1.84 \mathrm{~A}$ & $1.2 \mathrm{~B}$ & 0.25 & $44.4 \mathrm{~A}$ & 4.7 & 233 \\
\hline $\mathrm{CL}+\mathrm{MIN}$ & 2.6 & $0.176 \mathrm{~A}$ & $1.71 \mathrm{~B}$ & $1.3 \mathrm{AB}$ & 0.28 & $43.9 \mathrm{~A}$ & 4.7 & 212 \\
\hline $\mathrm{YW}+\mathrm{MIN}$ & 2.6 & $0.174 \mathrm{~A}$ & $1.84 \mathrm{~A}$ & $1.3 \mathrm{AB}$ & 0.26 & $43.1 \mathrm{AB}$ & 4.5 & 238 \\
\hline FGN & 2.5 & $0.165 \mathrm{~B}$ & $1.66 \mathrm{~B}$ & $1.4 \mathrm{~A}$ & 0.28 & $40.4 \mathrm{C}$ & 4.4 & 219 \\
\hline Treatment & NS & $* *$ & $* * *$ & $* *$ & NS & $* * *$ & NS & NS \\
\hline 2013 & $2.8 \mathrm{~A}$ & $0.199 \mathrm{~A}$ & $1.48 \mathrm{~B}$ & $1.59 \mathrm{~A}$ & $0.34 \mathrm{~A}$ & $45.6 \mathrm{~A}$ & $3.7 \mathrm{~B}$ & $137 \mathrm{C}$ \\
\hline 2014 & $2.5 \mathrm{~B}$ & $0.168 \mathrm{~B}$ & $2.26 \mathrm{~A}$ & $1.19 \mathrm{~B}$ & $0.22 \mathrm{C}$ & $44.1 \mathrm{~B}$ & $4.9 \mathrm{~A}$ & $230 \mathrm{~B}$ \\
\hline 2015 & $2.4 \mathrm{C}$ & $0.149 \mathrm{C}$ & $1.49 \mathrm{~B}$ & $1.21 \mathrm{~B}$ & $0.26 \mathrm{~B}$ & $38.1 \mathrm{C}$ & $5.0 \mathrm{~A}$ & $308 \mathrm{~A}$ \\
\hline Year & $* * *$ & $* * *$ & $* * *$ & $* * *$ & $* * *$ & $* * *$ & $* * *$ & $* * *$ \\
\hline Treatment $\times$ Year & NS & NS & NS & NS & NS & NS & NS & NS \\
\hline
\end{tabular}

${ }^{\mathrm{z}}$ Different letters within a column indicate significantly different means for the main effects (year and treatment) using Tukey's HSD test.

${ }^{\mathrm{y}}$ Measured as a percentage of leaf dry weight.

Ns, *,**,*** Nonsignificant or significant differences at $P \leq 0.05,0.01$, or 0.001 , respectively. 
Table 4. Plant available soil mineral concentration measured on soil samples (15-cm depth) taken $30 \mathrm{~cm}$ from the trunks of 'Red Delicious'/“M.26' trees in 2013 , 2014, and 2015 under the unfertilized control (CON) and six fertilizer treatments [calcium nitrate (MIN), chicken litter compost (CL), yard waste (YW) compost, integrated chicken litter compost and calcium nitrate (CL + MIN), integrated yard waste compost and calcium nitrate (YW + MIN), and fertigation $(\mathrm{FGN})]$ in Winchester, VA.

\begin{tabular}{|c|c|c|c|c|c|c|c|c|}
\hline & $\mathrm{P}\left(\mathrm{mg} \cdot \mathrm{kg}^{-1}\right)$ & $\mathrm{K}\left(\mathrm{mg} \cdot \mathrm{kg}^{-1}\right)$ & $\mathrm{Ca}\left(\mathrm{mg} \cdot \mathrm{kg}^{-1}\right)$ & $\mathrm{Mg}\left(\mathrm{mg} \cdot \mathrm{kg}^{-1}\right)$ & $\mathrm{B}\left(\mathrm{mg} \cdot \mathrm{kg}^{-1}\right)$ & $\mathrm{Cu}\left(\mathrm{mg} \cdot \mathrm{kg}^{-1}\right)$ & $\mathrm{Mn}\left(\mathrm{mg} \cdot \mathrm{kg}^{-1}\right)$ & $\mathrm{Zn}\left(\mathrm{mg} \cdot \mathrm{kg}^{-1}\right)$ \\
\hline$\overline{\mathrm{CON}}$ & & & $1,261 \mathrm{BC}^{\mathrm{z}}$ & & $0.575 \mathrm{~B}$ & $0.98 \mathrm{~A}$ & & $13 \mathrm{BC}$ \\
\hline MIN & & & $1,278 \mathrm{BC}$ & & $0.533 \mathrm{~B}$ & $0.84 \mathrm{AB}$ & & $10 \mathrm{C}$ \\
\hline CL & & & $1,861 \mathrm{~A}$ & & $1.000 \mathrm{~A}$ & $0.70 \mathrm{BC}$ & & $17 \mathrm{~A}$ \\
\hline YW & & & $1,807 \mathrm{~A}$ & & $1.017 \mathrm{~A}$ & $0.62 \mathrm{BC}$ & & $12 \mathrm{BC}$ \\
\hline $\mathrm{CL}+\mathrm{MIN}$ & & & $1,769 \mathrm{~A}$ & & $0.925 \mathrm{~A}$ & $0.65 \mathrm{BC}$ & & $16 \mathrm{AB}$ \\
\hline $\mathrm{YW}+\mathrm{MIN}$ & & & $1,654 \mathrm{AB}$ & & $0.833 \mathrm{~A}$ & $0.52 \mathrm{C}$ & & $12 \mathrm{BC}$ \\
\hline FGN & & & $1,173 \mathrm{C}$ & & $0.475 \mathrm{~B}$ & $0.88 \mathrm{AB}$ & & $11 \mathrm{C}$ \\
\hline Treatment & $* * *$ & $* * *$ & $* * *$ & $* * *$ & $* * *$ & $* * *$ & $* * *$ & $* * *$ \\
\hline 2013 & & & $1,510 \mathrm{~B}$ & & $0.739 \mathrm{~B}$ & $0.66 \mathrm{~B}$ & & $13 \mathrm{AB}$ \\
\hline 2014 & & & $1,361 \mathrm{~B}$ & & $0.657 \mathrm{~B}$ & $0.86 \mathrm{~A}$ & & $12 \mathrm{~B}$ \\
\hline 2015 & & & $1,758 \mathrm{~A}$ & & $0.900 \mathrm{~A}$ & $0.69 \mathrm{~B}$ & & $15 \mathrm{~A}$ \\
\hline Year & $* * *$ & NS & $* * *$ & $* * *$ & $* * *$ & $*$ & $* * *$ & $* *$ \\
\hline Treatment $\times$ Year & $* * *$ & $* *$ & NS & $* *$ & NS & NS & NS & $* *$ \\
\hline
\end{tabular}

Treatment effects within the Treatment $\times$ Year interaction:

\begin{tabular}{|c|c|c|c|c|c|}
\hline 2013 & $\mathrm{CON}$ & $22 \mathrm{~B}$ & $125 \mathrm{C}$ & $110 \mathrm{C}$ & $50 \mathrm{AB}$ \\
\hline 2013 & MIN & $20 \mathrm{~B}$ & $90 \mathrm{C}$ & $97 \mathrm{C}$ & $43 \mathrm{AB}$ \\
\hline 2013 & $\mathrm{CL}$ & $133 \mathrm{~A}$ & $144 \mathrm{BC}$ & $161 \mathrm{AB}$ & $39 \mathrm{~B}$ \\
\hline 2013 & YW & $36 \mathrm{~B}$ & $241 \mathrm{~A}$ & $172 \mathrm{~A}$ & $55 \mathrm{AB}$ \\
\hline 2013 & $\mathrm{CL}+\mathrm{MIN}$ & $110 \mathrm{~A}$ & $144 \mathrm{BC}$ & $152 \mathrm{AB}$ & $54 \mathrm{AB}$ \\
\hline 2013 & $\mathrm{YW}+\mathrm{MIN}$ & $33 \mathrm{~B}$ & $187 \mathrm{~B}$ & $139 \mathrm{ABC}$ & $58 \mathrm{~A}$ \\
\hline 2013 & FGN & $\begin{array}{l}17 \mathrm{~B} \\
* * *\end{array}$ & $\begin{array}{r}113 \mathrm{C} \\
* * *\end{array}$ & $\begin{array}{r}99 \mathrm{C} \\
* *\end{array}$ & $\begin{array}{c}49 \mathrm{AB} \\
* * *\end{array}$ \\
\hline 2014 & $\mathrm{CON}$ & $27 \mathrm{C}$ & $116 \mathrm{C}$ & $114 \mathrm{AB}$ & 15 \\
\hline 2014 & MIN & $18 \mathrm{C}$ & $77 \mathrm{C}$ & 92 B & 13 \\
\hline 2014 & CL & $92 \mathrm{~A}$ & $189 \mathrm{~B}$ & $152 \mathrm{AB}$ & 16 \\
\hline 2014 & YW & $27 \mathrm{C}$ & $247 \mathrm{~A}$ & $158 \mathrm{~A}$ & 19 \\
\hline 2014 & $\mathrm{CL}+\mathrm{MIN}$ & $69 \mathrm{~B}$ & $150 \mathrm{BC}$ & $133 \mathrm{AB}$ & 13 \\
\hline 2014 & $\mathrm{YW}+\mathrm{MIN}$ & $25 \mathrm{C}$ & $181 \mathrm{~B}$ & $127 \mathrm{AB}$ & 19 \\
\hline 2014 & FGN & $\begin{array}{l}14 \mathrm{C} \\
* * *\end{array}$ & $\begin{array}{r}89 \mathrm{C} \\
* * *\end{array}$ & $\begin{array}{r}88 \mathrm{~B} \\
* *\end{array}$ & $11_{\text {NS }}$ \\
\hline 2015 & $\mathrm{CON}$ & $22 \mathrm{C}$ & $101 \mathrm{CD}$ & $111 \mathrm{BCD}$ & $14 \mathrm{C}$ \\
\hline 2015 & MIN & $18 \mathrm{C}$ & $74 \mathrm{D}$ & $99 \mathrm{CD}$ & $15 \mathrm{C}$ \\
\hline 2015 & $\mathrm{CL}$ & $258 \mathrm{~A}$ & $217 \mathrm{~B}$ & $253 \mathrm{~A}$ & $24 \mathrm{AB}$ \\
\hline 2015 & YW & $50 \mathrm{C}$ & $326 \mathrm{~A}$ & $262 \mathrm{~A}$ & $35 \mathrm{~A}$ \\
\hline 2015 & $\mathrm{CL}+\mathrm{MIN}$ & $146 \mathrm{~B}$ & $165 \mathrm{C}$ & $179 \mathrm{~B}$ & $19 \mathrm{ABC}$ \\
\hline 2015 & $\mathrm{YW}+\mathrm{MIN}$ & $34 \mathrm{C}$ & $196 \mathrm{BC}$ & $160 \mathrm{BC}$ & $26 \mathrm{AB}$ \\
\hline 2015 & FGN & $\begin{array}{l}13 \mathrm{C} \\
* * *\end{array}$ & $\begin{array}{c}78 \mathrm{D} \\
* * *\end{array}$ & $\begin{array}{r}78 \mathrm{D} \\
* * *\end{array}$ & $\begin{array}{c}12 \mathrm{C} \\
* * *\end{array}$ \\
\hline
\end{tabular}

${ }^{\mathrm{z}}$ Different letters within a column indicate significantly different means for the main effects (year and treatment) or, for significant interactions, the treatment effects within each year using Tukey's HSD test.

ns, ${ }^{*}, * *,{ }^{* * *}$ Nonsignificant or significant differences at $P \leq 0.05,0.01$, or 0.001 , respectively.

(FisherBrand G6; Fisher, Waltham, MA). The incubated sample contained $10 \mathrm{~mL}$ of de-ionized water and extracted after $7 \mathrm{~d}$ at $30{ }^{\circ} \mathrm{C}$, at which time $2.67 \mathrm{M} \mathrm{KCl}$ was added before extraction. Samples were placed on an orbital shaker for $1 \mathrm{~h}$ at $3.3 \mathrm{r} \cdot \mathrm{s}^{-1}$, and centrifuged at $500 g_{\mathrm{n}}$ for $10 \mathrm{~min}$ before filtration. All samples were stored at $-20{ }^{\circ} \mathrm{C}$ until $\mathrm{NH}_{4}-\mathrm{N}$ and $\mathrm{NO}_{3}-\mathrm{N}$ concentrations were measured using a Lachat QuickChem 8500 Series 2 Flow Injection Analysis System (Loveland, CO). Ammonia was measured using protocol \#12-107-06-2-A, and $\mathrm{NO}_{3}{ }^{-}$ was measured using protocol \#12-107-04-1-B (Lachat Instruments, 2014).

Microbial biomass $\mathrm{C}$ was measured using the direct chloroform $\left(\mathrm{CHCl}_{3}\right)$ fumigation extraction method (Fierer and Schimel, 2003). Ten grams of soil from each sample and $40 \mathrm{~mL}$ of $0.05 \mathrm{~m}$ potassium sulfate $\left(\mathrm{K}_{2} \mathrm{SO}_{4}\right)$ were placed in two separate $70 \mathrm{~mL}$ glass vials with Teflon-lined lids. Fumigated samples received $0.5 \mathrm{~mL}$ of amylated $\mathrm{CHCl}_{3}$. Unfumigated samples did not receive $\mathrm{CHCl}_{3}$, but were otherwise treated the same as fumigated samples. Samples were shaken at $2.5 \mathrm{r} \cdot \mathrm{s}^{-1}$ on an orbital shaker for $4 \mathrm{~h}$ and allowed to settle for $30 \mathrm{~min}$ before being decanted into $50-\mathrm{mL}$ conical tubes. Samples were centrifuged for $10 \mathrm{~min}$ at 500 $g_{n}$ and filtered (FisherBrand G6; Fisher). The filtrate was sparged with compressed nitrogen gas for $20 \mathrm{~min}$ to remove any remaining $\mathrm{CHCl}_{3}$ from the solution and stored at $-20{ }^{\circ} \mathrm{C}$ until use. Blank samples with no soil were prepared in the same manner. Before analysis, samples were diluted 1:2(v:v) with deionized water. The total carbon was quantified using a Shimadzu (Columbia, MD) carbon analyzer model TOC-VCPH + TNM-1 with an auto-sampler, using high-temperature oxidation catalyzed with platinum-coated alumina beads (temperature $720{ }^{\circ} \mathrm{C}$ ) in nonpurgeable organic carbon mode (Bird et al., 2003). Nonpurgeable organic $\mathrm{C}$ was measured using a nondispersive infrared detector. Each sample was run in triplicate. Microbial biomass was calculated by multiplying the difference between the fumigated and unfumigated samples by the $k_{e c}$ value of 0.45 (Joergensen, 1996).
All data were analyzed using PROC GLIMMIX in SAS 9.4 (SAS Institute Inc., Cary, NC). For trunk cross-sectional area, leader growth, yield, and fruit quality/maturity data, Treatment was considered a fixed effect and block a random effect. Mean separation was determined using Tukey's honestly significant differences posthoc test at the $P \leq 0.05$ level. For flower cluster density, leaf minerals, soil minerals and chemical properties, PMN, and microbial biomass $\mathrm{C}$, Treatment, Year, and Treatment $\times$ Year were considered fixed effects, and block was a random effect. When interaction effects were significant, treatment effects were partitioned within each year using the LSMEANS values generated with the SLICEDIFF command and mean separation was determined using the least squares differences (LSD) test at the $P \leq 0.05$ level. Soil microbial respiration data were analyzed as a repeated measure using treatment as a fixed effect, and block and block $x$ treatment as random effects, and significance was determined using the LSD test at the $P \leq 0.05$ level. 
Table 5. Soil total carbon, total nitrogen, the $\mathrm{C}: \mathrm{N}$ ratio, cation exchange capacity (CEC), organic matter (OM), $\mathrm{pH}$, and soluble salts (SS) measured on soil samples (15-cm depth) taken $30 \mathrm{~cm}$ from the trunks of 'Red Delicious'/'M.26' trees in 2013, 2014, and 2015 under the unfertilized control (CON) and six fertilizer treatments [calcium nitrate (MIN), chicken litter compost (CL), yard waste (YW) compost, integrated chicken litter compost and calcium nitrate (CL + MIN), integrated yard waste compost and calcium nitrate ( $\mathrm{YW}+\mathrm{MIN})$, and fertigation (FGN)] in Winchester, VA.

\begin{tabular}{|c|c|c|c|c|c|c|c|}
\hline & $\mathrm{OM}\left(\mathrm{g} \cdot \mathrm{kg}^{-1}\right)$ & $\mathrm{C}\left(\mathrm{g} \cdot \mathrm{kg}^{-1}\right)$ & $\mathrm{N}\left(\mathrm{g} \cdot \mathrm{kg}^{-1}\right)$ & $\mathrm{C}: \mathrm{N}$ & $\mathrm{CEC}\left(\mathrm{meq} \cdot 100 \mathrm{~g}^{-1}\right)$ & $\mathrm{pH}$ & $\mathrm{SS}\left(\mathrm{mmhos} \cdot \mathrm{cm}^{-1}\right)$ \\
\hline$\overline{\mathrm{CON}}$ & & & $2.1 \mathrm{C}^{\mathrm{z}}$ & 6.91 & $7.8 \mathrm{BC}$ & $6.6 \mathrm{AB}$ & 0.2 \\
\hline MIN & & & $2.3 \mathrm{BC}$ & 6.42 & $7.5 \mathrm{C}$ & $6.6 \mathrm{AB}$ & 0.38 \\
\hline $\mathrm{CL}$ & & & $2.8 \mathrm{BC}$ & 9.11 & $11.4 \mathrm{~A}$ & $6.8 \mathrm{~A}$ & 0.35 \\
\hline YW & & & $3.4 \mathrm{~A}$ & 9.45 & $11.4 \mathrm{~A}$ & $6.9 \mathrm{~A}$ & 0.36 \\
\hline $\mathrm{CL}+\mathrm{MIN}$ & & & $2.6 \mathrm{BC}$ & 8.88 & $10.5 \mathrm{~A}$ & $6.9 \mathrm{~A}$ & 0.35 \\
\hline $\mathrm{YW}+\mathrm{MIN}$ & & & $2.9 \mathrm{AB}$ & 8.85 & $9.8 \mathrm{AB}$ & $6.9 \mathrm{~A}$ & 0.34 \\
\hline FGN & & & $2.3 \mathrm{BC}$ & 9.03 & $7.3 \mathrm{C}$ & $6.3 \mathrm{~B}$ & 0.4 \\
\hline Treatment & $* * *$ & $* * *$ & $* * *$ & NS & $* * *$ & $* *$ & NS \\
\hline 2013 & & & $2.9 \mathrm{~B}$ & $6.06 \mathrm{~B}$ & $9.2 \mathrm{~B}$ & $6.6 \mathrm{~B}$ & 0.39 \\
\hline 2014 & & & $1.6 \mathrm{C}$ & $12.34 \mathrm{~A}$ & $8.4 \mathrm{~B}$ & $6.7 \mathrm{AB}$ & 0.31 \\
\hline 2015 & & & $3.3 \mathrm{~A}$ & $6.74 \mathrm{~B}$ & $10.7 \mathrm{~A}$ & $6.9 \mathrm{~A}$ & 0.36 \\
\hline Year & $* *$ & $*$ & $* * *$ & $* * *$ & $* * *$ & $* *$ & NS \\
\hline Treatment $\times$ Year & $*$ & $*$ & NS & NS & NS & NS & NS \\
\hline
\end{tabular}

Treatment effects within the Treatment $\times$ Year interaction:

$\begin{array}{llll}2013 & \mathrm{CON} & 29 \mathrm{~B} & 13.7 \mathrm{~B} \\ 2013 & \mathrm{MIN} & 31 \mathrm{~B} & 14.3 \mathrm{~B} \\ 2013 & \mathrm{CL} & 38 \mathrm{~A} & 17.8 \mathrm{~B} \\ 2013 & \mathrm{YW} & 47 \mathrm{~A} & 28.5 \mathrm{~A} \\ 2013 & \mathrm{CL}+\mathrm{MIN} & 43 \mathrm{~A} & 17.0 \mathrm{~B} \\ 2013 & \mathrm{YW}+\mathrm{MIN} & 46 \mathrm{~A} & 24.1 \mathrm{AB} \\ 2013 & \mathrm{FGN} & 29 \mathrm{~B} & 13.8 \mathrm{~B} \\ & & * & * * \\ 2014 & \mathrm{CON} & 28 \mathrm{AB} & 14.6 \mathrm{AB} \\ 2014 & \mathrm{MIN} & 22 \mathrm{~B} & 12.2 \mathrm{~B} \\ 2014 & \mathrm{CL} & 29 \mathrm{AB} & 21.6 \mathrm{AB} \\ 2014 & \text { YW } & 39 \mathrm{~A} & 20.7 \mathrm{AB} \\ 2014 & \mathrm{CL}+\mathrm{MIN} & 39 \mathrm{~A} & 24.1 \mathrm{~A} \\ 2014 & \text { YW }+\mathrm{MIN} & 35 \mathrm{~A} & 19.8 \mathrm{AB} \\ 2014 & \mathrm{FGN} & 23 \mathrm{~B} & 20.7 \mathrm{AB} \\ & & * & * * \\ 2015 & \mathrm{CON} & 24 \mathrm{C} & 12.6 \mathrm{C} \\ 2015 & \mathrm{MIN} & 26 \mathrm{C} & 14.6 \mathrm{C} \\ 2015 & \mathrm{CL} & 44 \mathrm{~B} & 29.9 \mathrm{~B} \\ 2015 & \text { YW } & 61 \mathrm{~A} & 46.1 \mathrm{~A} \\ 2015 & \mathrm{CL}+\mathrm{MIN} & 38 \mathrm{~B} & 22.1 \mathrm{BC} \\ 2015 & \text { YW }+\mathrm{MIN} & 39 \mathrm{~B} & 28.4 \mathrm{~B} \\ 2015 & \text { FGN } & 24 \mathrm{C} & 12.2 \mathrm{C} \\ & & * * * & * * *\end{array}$

${ }^{\mathrm{z}}$ Different letters within a column indicate significantly different means for the main effects (year and treatment) or, for significant interactions, the treatment effects within each year using Tukey's HSD test.

Ns, $*, * * * * *$ Nonsignificant or significant differences at $P \leq 0.05,0.01$, or 0.001 , respectively.

\section{Results}

No differences in TCSA or leader growth were observed during any year of our study and by the end of the 3 years there were no differences in tree size (Table 2). Red peel color was the only fruit quality or maturity parameter affected by the treatments. In 2015, fruit yield was $68 \%, 53 \%$, and $59 \%$ greater from the $\mathrm{CON}$, MIN, and FGN trees, respectively, than the CL trees. Fruit from trees fertilized with MIN had on average $3 \%$ greater red peel color than fruit from FGN trees, but all treatments had fruit with greater than $92 \%$ red blush. Flower cluster density ranged between 6 and 9 blossoms $/ \mathrm{cm}^{2}$ TCSA and did not differ among treatments in 2015. In 2016, flower cluster density ranged between 8 and 14 blossoms $/ \mathrm{cm}^{2}$ TCSA. The MIN and YW treatments had significantly greater flower cluster density than the CL treatment (data not shown).

There were no detectable differences in leaf $\mathrm{N}$ concentration among the treatments, but there was an overall reduction in $\mathrm{N}$ leaf content from 2013 to 2015 (Table 3). Leaf P concentration was greater the CL, CL + MIN, and YW + MIN treatments than the FGN treatment; however, none of the treatments had greater $\mathrm{P}$ relative to CON. The YW and YW + MIN treatments had greater leaf $\mathrm{K}$ concentrations than the $\mathrm{CON}$, MIN, CL + MIN, and FGN treatments. The FGN and MIN treatments had greater leaf $\mathrm{Ca}$ concentration than the YW treatment, but not compared with CON. All compost and compost + mineral fertilizer treatments had greater leaf $\mathrm{B}$ concentrations compared with the $\mathrm{CON}$ and FGN treatments.

Over the course of this study, the CL and $\mathrm{CL}+\mathrm{MIN}$ treatments had greater extractable soil $\mathrm{P}$ than the other treatments, though there was some overall year-to-year variability in the amount of $P$ that was measured (Table 4). In 2014 and 2015, CL had greater extractable soil $\mathrm{P}$ content than CL + MIN. All of the compost and compost + mineral treatments had greater extractable soil $\mathrm{K}$ than the $\mathrm{CON}, \mathrm{MIN}$, and FGN treatments, but there was some yearto-year variability among the compost treatments. By 2015, the YW treatment had the greatest extractable soil $\mathrm{K}$ concentration while CON, MIN, and FGN had the lowest. The CL, $\mathrm{CL}+\mathrm{MIN}$, and $\mathrm{YW}$ treatments had greater extractable soil $\mathrm{Ca}$ than the CON, MIN, and FGN treatments.

The compost and compost + mineral treatments had greater extractable soil $\mathrm{Mg}$ and $\mathrm{B}$ than the $\mathrm{CON}, \mathrm{MIN}$, and FGN treatments. The CON had similar extractable soil $\mathrm{Cu}$ concentration to the MIN and FGN treatments and a greater concentration than the four treatments with compost. There were small or no differences for soil extractable Mn in 2013 and 2014, but the CL, YW, and $\mathrm{YW}+\mathrm{MIN}$ treatments had greater $\mathrm{Mn}$ concentration in 2015 than the CON, MIN, and FGN treatments. The CL treatments had a greater extractable soil $\mathrm{Zn}$ concentration than the other treatments, though the $\mathrm{CL}+$ MIN treatment was similar to the CON, YW, and $\mathrm{YW}+\mathrm{MIN}$ treatments.

In 2013 and 2015, the CL, YW, CL + MIN, and $\mathrm{YW}+\mathrm{MIN}$ treatments had greater soil OM than the CON, MIN and FGN treatments (Table 5). In 2014, the YW, CL + MIN, and $\mathrm{YW}+\mathrm{MIN}$ had a greater OM content than MIN and FGN, with the CON and CL treatments being intermediate. In 2015, the CL, YW, $\mathrm{CL}+\mathrm{MIN}$, and YW + MIN treatments had 

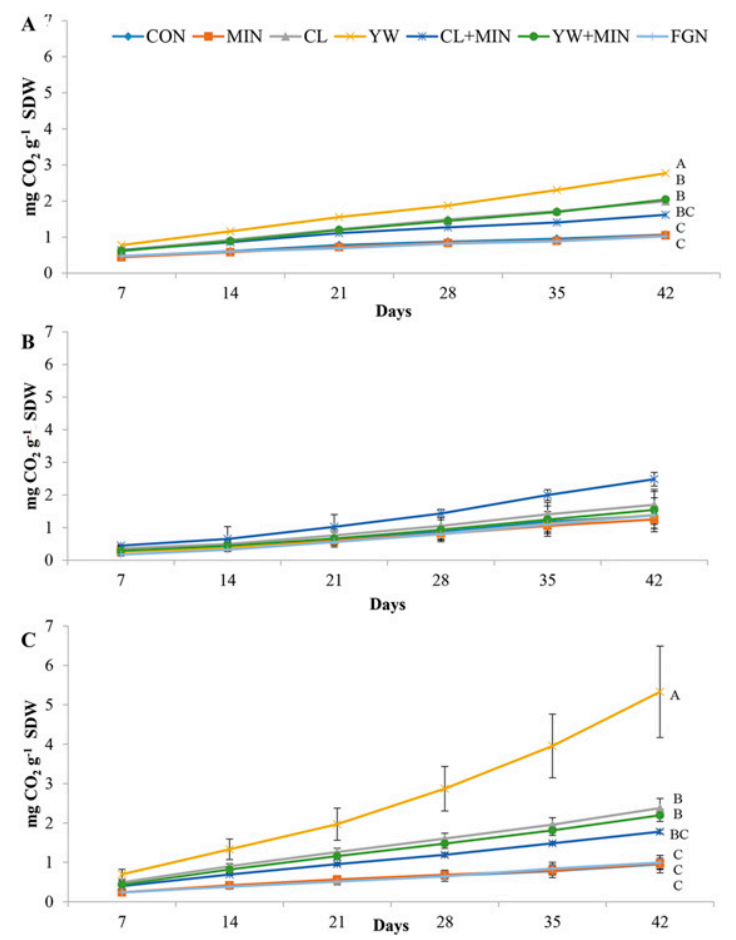

Fig. 1. Cumulative soil microbial respiration [reported as the amount of carbon dioxide $\left(\mathrm{CO}_{2}\right)$ captured weekly on a soil dry weight basis (SDW)] in 2013 (A), 2014 (B), and 2015 (C). Data were measured on soil samples (15-cm depth) taken $30 \mathrm{~cm}$ from the trunks of 'Red Delicious'/'M.26' trees under the unfertilized control (CON) and six fertilizer treatments [calcium nitrate (MIN), chicken litter compost (CL), yard waste (YW) compost, integrated chicken litter compost and calcium nitrate (CL + MIN), integrated yard waste compost and calcium nitrate (YW + MIN), and fertigation (FGN)] in Winchester, VA. Data were analyzed as repeated measures. Different letters indicate significantly different means at $P \leq 0.05$ using the least significant differences test $(n=4)$. Error bars are SE.

$45 \%, 61 \%, 37 \%$, and $38 \%$ more OM than the CON soil, respectively. By 2015, the CL, YW, $\mathrm{CL}+\mathrm{MIN}$, and $\mathrm{YW}+\mathrm{MIN}$ treatments also had greater $\mathrm{C}$ concentration than the $\mathrm{CON}$, MIN and FGN treatments. Throughout this study, total soil $\mathrm{N}$ was greater in the YW and $\mathrm{YW}+\mathrm{MIN}$ treatments than the CON, but the other treatments had similar $\mathrm{N}$ concentrations. There were no treatment differences in the $\mathrm{C}: \mathrm{N}$ ratio among the treatments. The $\mathrm{CL}$, $\mathrm{YW}$, and $\mathrm{CL}+\mathrm{MIN}$ treatments had greater CEC than the CON, MIN, and FGN treatments, and the YW + MIN was intermediate. The CL, YW, CL + MIN, and YW + MIN treatments had a less acidic soil than the FGN treatment. There were no differences among the treatments for the soluble salt content.

In 2013 and 2015, the fertilizer treatments affected soil microbial respiration, but not in 2014 (Fig. 1). Soil fertilized with YW had greater microbial respiration rates than all other treatments in $2013(P=$ $0.0006)$ and $2015(P<0.0001)$. Soil microbial respiration was 2.5 times greater in $\mathrm{YW}$ fertilized soil than in the CON soils in 2013, and 5 times greater in 2015. Soil microbial respiration was greatest in the $\mathrm{YW}, \mathrm{CL}$, and YW + MIN soils (Fig. 1). On average, PMN was $17 \mu \mathrm{g} \cdot \mathrm{g}^{-1} \pm 1.7$ and microbial biomass carbon was $928 \mu \mathrm{g} \mathrm{C} / \mathrm{g} \mathrm{SDW} \pm 54$, but there were no differences among treatments (data not shown).

\section{Discussion}

During the first 3 years of apple orchard establishment, none of the fertilizer treatments increased tree growth or leaf $\mathrm{N}$ status compared with the unfertilized control (CON). However, within three months after the initial fertilizer application, the compost treatments increased soil mineral content and $\mathrm{OM}$ and these changes persisted through the length of the study. Our results are similar to other studies in that we demonstrated that compost, integrated compost-mineral fertilizer, or fertigation applications may not always increase orchard productivity in young orchards, but these treatments can affect soil quality and therefore the potential long-term productivity and environmental impacts of the orchard system (Forge et al., 2013; Kramer et al., 2006; Neilsen et al., 2004, 2009; Sas-Paszt et al., 2014; Yao et al., 2006). The integrated $\mathrm{CL}+\mathrm{MIN}$ and YW + MIN treatments, which we hypothesized would supply apple trees with sufficient nitrogen to maintain adequate growth and productivity while also improving soil quality, did not increase tree growth or leaf $\mathrm{N}$ more so than the other treatments or the control. Fine-textured soils with higher OM content, like those in the Shenandoah Valley of Virginia where our research took place, mineralize more $\mathrm{N}$ than soils with lower OM content, resulting in increased plant productivity (Bauer and Black, 1994). Trees in our study were likely supplied with adequate $\mathrm{N}, \mathrm{P}$, and $\mathrm{K}$ nutrition from existing soil $\mathrm{OM}$, thus the addition of fertilizers, in the form of mineral $\mathrm{N}$ or as compost may have not been necessary for this orchard.

Composts, regardless of feedstock, soil type, or crop, have been reported to enhance edaphic factors, such as OM, microbial activity, and soil mineral concentration, including $\mathrm{P}$ and $\mathrm{K}$, in orchard systems (Baldi et al., 2010; Forge et al., 2013; Kramer et al., 2006). Despite significant increases in OM and total C in 2013 and 2015, neither CL nor YW increased PMN or microbial biomass carbon. These results were unexpected because soil OM and carbon additions typically increase microbial biomass carbon and microbial activity (Schnurer et al., 1985; Wardle, 1992). Peck et al. (2011) demonstrated that additions of $\mathrm{OM}$ in the form of wood chips to the orchard floor increased microbial biomass carbon, but additions of composted chicken litter did not, indicating that the specific $\mathrm{C}: \mathrm{N}$ ratio of the amendment influences microbial biomass. Kramer et al. (2006) found that compost and calcium nitrate $\left[\mathrm{Ca}\left(\mathrm{NO}_{3}\right)_{2}\right]$ increased soil $\mathrm{OM}$, but did not affect microbial biomass or potentially mineralizable $\mathrm{N}$, compared with mineral fertilizer application in an established apple orchard planted on coarse, sandy soil in an arid environment. In our study, soil microbial respiration increased in compostamended soils compared with CON, MIN, and FGN treatments in 2013 and 2015 indicating that soil microbes used the added $\mathrm{C}$ to sustain their populations throughout the duration of the experiment. Soil microbes mineralize $\mathrm{N}$ and $\mathrm{P}$ from $\mathrm{OM}$ slowly throughout the growing season, potentially leading to increased leaf mineral nutrient content in future years.

Applications of the compost and integrated compost-mineral $\mathrm{N}$ fertilizers increased soil $\mathrm{P}, \mathrm{K}$, and $\mathrm{B}$ concentration, which led to increased leaf concentrations of these minerals. However, greater leaf $\mathrm{P}$, $\mathrm{K}$, and $\mathrm{B}$ concentrations were not associated with increased tree growth, flower cluster density, or fruit yield or quality during this study. In addition, increased soil $\mathrm{Mn}$ and $\mathrm{Zn}$ concentrations in the compost treatments did not increase leaf concentrations of these minerals. Leaf $\mathrm{Mn}$ and $\mathrm{Zn}$ concentrations were above the adequate level for all treatments, but $\mathrm{Ca}$ and $\mathrm{Mg}$ leaf concentrations were deficient among all treatments, indicating that in the short-term compost may not provide sufficient $\mathrm{Ca}$ and $\mathrm{Mg}$ to overcome these deficiencies. Calcium is somewhat mobile in the soil; however, in apple trees, translocation of $\mathrm{Ca}$ occurs very slowly (Vang-Petersen, 1980). Although Mg is readily translocated in plants, $\mathrm{Mg}$ deficiencies commonly occur because of reduced mobility, long term soil depletion, or unbalanced fertilization practices that significantly increase soil K (Gransee and Fuhrs, 2013). Thus, foliar applications of $\mathrm{Ca}$ and $\mathrm{Mg}$ would likely be necessary in commercial orchards to prevent common nutrient disorders regardless 
of the $\mathrm{N}$ fertilizer being used (Vang-Petersen, 1980).

In summary, the fertilizer treatments and rates used in our experiment did not affect leaf $\mathrm{N}$ or tree growth and only minimally affected fruit yield and quality within the first 3 years after planting the orchard. Because unnecessary applications of $\mathrm{N}$ fertilizer in orchards can lead to reduced fruit quality and $\mathrm{N}$ leaching, these results suggest that apple growers should carefully consider site history, leaf mineral content, soil texture, and OM before making fertilizer applications in young orchards. The lack of treatment differences in our experiment for tree growth and fruit quality were likely due to adequate soil nutritional status and OM content before fertilizer applications. However, compost increased soil properties that are associated with long-term soil fertility, such as OM, soil $\mathrm{C}$, and microbial respiration. It is possible that these increases will positively impact orchard productivity in future years. Increased soil mineral nutrition, OM, CEC, and microbial activity from compost may also reduce the need for future applications of N, P, and K. However, longer-term studies are needed to understand the effects of these fertilization practices as the orchard matures and reaches full productivity.

\section{Literature Cited}

Baldi, E., M. Toselli, G. Marcolini, M. Quartieri, E. Cirillo, A. Innocenti, and B. Marangoni. 2010. Compost can successfully replace mineral fertilizers in the nutrient management of commercial peach orchard. Soil Use Mgt. 26:346-353.

Bauer, A. and A.L. Black. 1994. Quantification of the effect of soil organic matter content on soil productivity. Soil Sci. Soc. Amer. J. 58:185-193.

Bird, S.M., M.S. Fram, and K.L. Crepeau. 2003. Method of analysis by the U.S. Geological Survey California District Sacramento LaboratoryDetermination of dissolved organic carbon in water by high temperature catalytic oxidation, method validation, and quality-control practices. High Temp. Open file report 03-366.

Blanpied, G.D. and K.J. Silsby. 1992. Predicting harvest date windows for apples. Cornell Coop. Ext., Geneva, NY. Info. Bul. 221.

Campbell-Nelson, K. 2015. Compost calculations. Univ. MA Coop. Ext. Amherst, MA. 10 June 2017. <ag.umass.edu/vegetable>.

Dong, S., L. Cheng, C.F. Scagel, and L.H. Fuchigami. 2005a. Method of nitrogen application in summer affects plant growth and nitrogen uptake in autumn in young Fuji/M.26 apple trees. Commun. Soil Sci. Plant Anal. 36:1465-1477.

Dong, S., D. Neilsen, G.H. Neilsen, and L.H. Fuchigami. 2005b. Foliar N application reduces soil $\mathrm{NO}_{3}{ }^{-} \mathrm{N}$ leaching loss in apple orchards. Plant Soil 268:357-366.

Fierer, N. and J.P. Schimel. 2003. A proposed mechanism for the pulse in carbon dioxide production commonly observed following the rapid rewetting of dry soil. Soil Sci. Soc. Amer. J. 67:798-805.

Forge, T., G. Neilsen, D. Neilsen, E. Hogue, and D. Faubion. 2013. Composted dairy manure and alfalfa hay mulch affect soil ecology and early production of 'Braeburn' apple on M.9 rootstock. HortScience 38:645-651.

Gransee, A. and H. Fuhrs. 2013. Magnesium mobility in soil as a challenge for soil and plant analysis, magnesium fertilization and root uptake under adverse growth conditions. Plant Soil 368:5-21.

Hartz, T.K., J.P. Mitchell, and C. Giannini. 2000. Nitrogen and carbon mineralization dynamics of manures and composts. HortScience 35: 209-212.

Hernández, T., C. Chocano, J. Moreno, and C. Garcia. 2014. Towards a more sustainable fertilization: Combined use of compost and inorganic fertilization for tomato cultivation. Agr. Ecosystem and Environ. 196:178-184.

Horneck, D.A. and R.O. Miller. 1998. Determination of total nitrogen in plant tissue, p. 37-51. In: Y.P. Kalra (ed.). Handbook and reference methods for plant analysis. CRC Press, New York, NY.

Joergensen, R.G. 1996. The fumigation-extraction method to estimate soil microbial biomass: Calibration of the $k_{e c}$ value. Soil Biol. Biochem. 28:25-31.

Kipp, J.A. 1992. Thirty years of fertilization and irrigation in Dutch apple orchards: A review. Fert. Res. 32:149-156.

Klein, I., I. Levin, B. Bar-Yosef, R. Assaf, and A. Berkovitz. 1989. Drip nitrogen fertigation of 'Starking Delicious' apple trees. Plant Soil 119:305-314.

Kramer, S.B., J.P. Reganold, J.D. Glover, B.J.M Bohannan, and H.A. Mooney. 2006. Reduced nitrate leaching and enhanced denitrifier activity and efficiency in organically fertilized soils Proc. Natl. Acad. Sci. USA 103:4522-4527.

Lachat Instruments. 2014. Methods list for automated ion analyzers. Loveland, CO.

Merwin, I.A., J.A. Ray, T.S. Steenhuis, and J. Boll. 1996. Groundcover management systems influence fungicide and nitrate- $\mathrm{N}$ concentrations in leachate and runoff from a New York apple orchard. J. Amer. Soc. Hort. Sci. 121:249-257.

Miller, R.O. 1998. High-temperature oxidation: Dry ashing, p. 53-57. In Y.P. Kalra (ed.) Handbook and Reference Methods for Plant Analysis. CRC Press, New York, NY.

Mylavarapu, R.S. and G.M. Zinati. 2009. Improvement of the soil properties using compost for optimum parsley production in sandy soils. Sci. Hort. 120:426-430.

Natural Resource Conservation Service (NRCS). 2001 U.S. Geological Survey, Frederick County, Virginia. 5 Aug. 2016. <http://websoilsurvey.sc.egov. usda.gov/>

Neilsen, D. and G.H. Neilsen. 2002. Efficient use of nitrogen and water in high-density apple orchards. HortTechnology 12:19-25.

Neilsen, G., T. Forge, D. Angers, D. Neilsen, and E. Hogue. 2014. Suitable orchard floor management strategies in organic apple orchards that augment soil organic matter and maintain tree performance. Plant Soil 378:325-335.
Neilsen, G.H., D. Neilsen, and L. Herbert. 2009. Nitrogen fertigation concentration and timing of application affects nitrogen nutrition, yield, firmness, and color of apples grown at high density. HortScience 44:1425-1431.

Neilsen, G.H., D. Neilsen, L.C. Herbert, and E.J. Hogue. 2004. Response of apple to fertigation of $\mathrm{N}$ and $\mathrm{K}$ under conditions susceptible to the development of K deficiency. J. Amer. Soc. Hort. Sci. 129:26-31.

Peck, G.M., I.A. Merwin, J.E. Thies, R.R. Schindelbeck, and M.G. Brown. 2011. Soil properties change during the transition to integrated and organic apple production in a New York orchard. Appl. Soil Ecol. 48:18-30.

Pfeiffer, D.G, J.C. Bergh, G.M. Peck, K.S. Yoder, J.F. Derr, M.J. Weaver, J. Parkhurst, D.L. Frank, A.R. Biggs, J.B. Kotcon, R.S. Chandran, C.R.R. Hooks, C.S. Walsh, and A. Brown. 2015. Virginia-West Virginia-Maryland Commercial Tree Fruit Spray Bulletin. VirginiaWest Virginia extension publication 456-419.

Robinson, T., A. Lakso, D. Greene, and S. Hoying. 2013. Precision crop load management. NY Fruit Qrtly. 21:3-9.

Rodella, A.A. and L.V. Saboya. 1999. Calibration of conductimetric determination of carbon dioxide. Soil Biol. Biochem. 31:2059-2060.

Rumberger, A., S. Yao, I.A. Merwin, E.B. Nelson, and J. Thies. 2004. Rootstock genotype and orchard replant position rather than soil fumigation or compost amendment determine tree growth and rhizosphere bacterial community composition in an apple replant soil. Plant Soil 264:247-260.

Sas-Paszt, L., K. Pruski, E. Żurawicz, B. Sumorok, E. Derkowska, and S. Gluszek. 2014. The effect of organic mulches and mycorrhizal substrate on growth, yield and quality of Gold Millennium apples on M.9 rootstock. Can. J. Plant Sci. 94:281-291.

Schnurer, J., M. Clarholm, and T. Rosswall. 1985. Microbial biomass and activity in an agricultural soil with different organic matter contents. Soil Biol. Biochem. 17:611-618.

Stamatiadis, S., M. Werner, and M. Buchanan. 1999. Field assessment of soil quality as affected by compost and fertilizer application in a broccoli field (San Benito County, California). Appl. Soil Ecol. 12:217-225.

United States Department of Agriculture and United States Composting Council. 2002. Test methods for the examination of composting and compost.

United State Environmental Protection Agency. 2010. Chesapeake Bay TMDL Executive Summary. U.S. EPA, Washington, D.C.

Vang-Petersen, O. 1980. Calcium nutrition of apple trees: A review. Sci. Hort. 12:1-9.

Wardle, D.A. 1992. A comparative assessment of factors which influence microbial biomass carbon and nitrogen levels in soil. Biol. Rev. Camb. Philos. Soc. 67:321-358.

Yao, S., I.A. Merwin, G.S. Abawi, and J.E. Thies. 2006. Soil fumigation and compost amendment alter soil microbial community composition but do not improve tree growth or yield in an apple replant site. Soil Biol. Biochem. $38: 587-599$. 